Bangl. J. Vet. Med. (2010). 8(1): 81 - 86

\title{
ANATOMICAL MEASUREMENTS FOR THE BLOCKING SITES OF PARAVERTEBRAL REGIONAL ANAESTHESIA IN BLACK BENGAL DOE
}

\author{
S. Sikder ${ }^{1}$, S.S.U. Ahmed ${ }^{1}$, A.S.M.G. Kibria ${ }^{2}$, M. S. Pallab ${ }^{1}$, M. B. Uddin ${ }^{3 *}$, J. Basu ${ }^{2}$ and M. M .Uddin ${ }^{2}$ \\ ${ }^{1}$ Department of Medicine and Surgery, ${ }^{2}$ Department of Anatomy and Histology, Chittagong Veterinary and \\ Animal Sciences University, Chittagong-4202. Bangladesh \\ ${ }^{3}$ Department of Medicine and Surgery, Sylhet Agricultural University, Bangladesh
}

\begin{abstract}
The study was conducted in 25 black Bengal does to determine the exact site of infiltration of local anaesthetics for the last thoracic (T13), first lumbar (L1) and second lumbar (L2) paravertebral spinal nerve block at the Dept. of Anatomy and Histology, Chittagong Veterinary and Animal Sciences University, Chittagong. The animals were anaesthetized with pentobarbital sodium @ $20 \mathrm{mg} / \mathrm{kg}$ body weight IM and bled to death by giving incision on the right common carotid artery and preserved by $10 \%$ formalin and dissected carefully to visualize the spinal nerves for the determination of the exact site of infiltration of local anaesthetic at the perineural area for paravertebral nerve block. For the vertical or proximal approach, the T13 spinal nerve was located at $0.8 \pm 0.17 \mathrm{~cm}$ caudally from head of the last rib, $0.7 \pm 0.22 \mathrm{~cm}$ cranio-medially from craniolateral angle of L1 transverse process and $3.4 \pm 0.20 \mathrm{~cm}$ laterally from dorsal midline; $\mathrm{L} 1$ spinal nerve was placed at $1.2 \pm 0.40$ $\mathrm{cm}$ medially from cranio-lateral angle of L2 transverse process and $3.5 \pm 0.17 \mathrm{~cm}$ laterally from the dorsal midline; and L2 spinal nerve was situated at $1.6 \pm 0.26 \mathrm{~cm}$ medially from cranio-lateral angle of L3 transverse process and $3.7 \pm 0.14 \mathrm{~cm}$ laterally from the dorsal midline. In horizontal or distal approach, T13 spinal nerve was located at $0.5 \pm 0.10 \mathrm{~cm}$ and $1.8 \pm$ $0.63 \mathrm{~cm}$ cranially from the cranio-lateral and caudo-lateral angles of L1 transverse process respectively and $4.6 \pm 0.30 \mathrm{~cm}$ laterally from dorsal midline at lateral border of L1 transverse process; L1 spinal nerve was placed at $0.4 \pm 0.10 \mathrm{~cm}$ and $1.4 \pm$ $0.28 \mathrm{~cm}$ caudally and cranially from the cranio-lateral and caudo-lateral angle of L2 transverse process respectively and $5.1 \pm$ $0.20 \mathrm{~cm}$ laterally from dorsal midline at lateral border of L2 transverse process and L2 spinal nerve was situated at $2.5 \pm 0.42$ $\mathrm{cm}$ and $0.5 \pm 0.10 \mathrm{~cm}$ caudally and cranially from the cranio-lateral and caudo-lateral angle of L3 transverse process respectively and $5.5 \pm 0.28 \mathrm{~cm}$ laterally from dorsal midline at lateral border of L3 transverse process. These measurements will be helpful to determine of the exact site for the deposition of the local anaesthetic agent at the perineural area of the specific paravertebral spinal nerve during paravertebral regional anaesthesia in doe.
\end{abstract}

Key words: Black Bengal, flank, paravertebral anaesthesia

\section{INTRODUCTION}

Regional anaesthesia is the first choice of anaesthesia in ruminants since general anaesthesia has certain limitations, anatomical and physiological peculiarities (Shokry, 1982) and regurgitation (Hossain, 1984; Hashim and Hossain, 1989).

In ruminants, flank region is the most common site for any laparotomy; caesarian section, rumenotomy, intestinal obstruction, volvulus, ruminal fistula, foreign body syndrome, hernia etc. (Lee, 2006; Kumar, 2003). This region is innervated by the last thoracic (T13), first lumbar (L1) and second lumbar (L2) spinal nerves (Getty, 1975).

The nerves innervated to the specific region are anaesthetized in regional anaesthesia (Lee, 2006). In caprine, T13, L1 and L2 spinal nerves are necessary to block for operations carried out through flank region (Hall and Clarke, 1989).

During regional anaesthesia of the flank region, nerves innervated to that region are blocked through injection of local anaesthetic as they emerge from the vertebral canal throuth the intervertebral foraminae in proximal paravertebral nerve block (Lee, 2006) or more distally at free ends of the lumbar transverse process and posterior border of the head of the last rib during distal paravertebral nerve block (Kumar, 2003).

Paravertebral nerve block results effective analgesia in all layers of the abdominal wall while the tissue infiltration, inverted L block may not produce analgesia of all muscle layers as well as peritoneum specially in fat animals (Sloss and Dufty, 1977). The paravertebral analgesia in goat is not always successful and unreliable in conventional techniques because variations in the course of dorsal and ventral branches of lumbar spinal nerves (Roe, 1986), divergences in the techniques and variations in the measurements from the landmarks (Cakala, 1961; Kumar, 2003; Hall and Clarke, 1989).

*Corresponding author: e-mail: bashir_vet@yahoo.com 


\section{S. Sikder and others}

However, paravertebral anaesthesia is easy to carry out and almost always effective if the anaesthesiologist has proper topographic anatomical knowledge of the courses of the T13, L1 and L2 nerves and finally exact location of these nerves from the landmarks innervated to the flank region (Shokry, 1982; Lee, 2006).

Techniques of the paravertebral nerve block have been carried out in cattle (Venugopalan, 2000; Hall and Clarke, 1989; Sharma, 2005; Riebold et al., 1982) but a few report on the variation in techniques and measurements for performing paravertebral regional anaesthesia in different breeds of goat (Constantinescu, 2001; Kumar, 2003; Shokry, 1982). Therefore, the present study was undertaken to determine the exact sites for infiltration of local anaesthetic agent for the blocking of T13, L1 and L2 spinal nerves from the specific landmarks during paravertebral regional anaesthesia in black Bengal doe.

\section{MATERIALS AND METHODS}

The research work was carried out on 25 black Bengal does (Capra hircus) at the Department of Anatomy and Histology, Faculty of Veterinary Medicine, Chittagong Veterinary and Animal Sciences University, Khulshi, Chittagong during the period from January to June 2009. All the does were adult (2-3 years), body weight 15 to $20 \mathrm{~kg}$, apparently healthy and devoid of any external abnormalities.

The animals were maintained in a well ventilated room and kept under good hygienic condition, allowed to graze in the open field for six hours everyday and also supplied concentrate mixture and water ad libitum.

The does were anaesthetized with pentobarbital sodium @ 20 mg / kg body weight IM and bled to death by giving incision on the right common carotid artery, after that the whole vascular system was flashed with $0.85 \%$ physiological saline solution and injected with $10 \%$ formalin for well preservation following the same route.

Careful dissection was made with the help of scalpel, scissors and forceps without rupture of the dorsal and ventral branches of the last thoracic (T13), first (L1) and second (L2) lumbar spinal nerves (Fig.1). Both the branches were made distinct by gentle separation of surrounding structures using tissue forceps. The exact location of the last thoracic, first and second lumbar spinal nerves were measured from the specific landmarks in metric rule during the course of dissection and the result were presented as mean \pm SD in Table 1 and 2 . Photographs were taken during the course of dissection. The measurement of the exact location from the landmarks of the T13, L1 and L2 spinal nerves for the proximal or vertical and distal or horizontal approaches of the paravertebral regional anaesthesia in black Bengal doe was described below and shown in the Fig. 2 and 3.

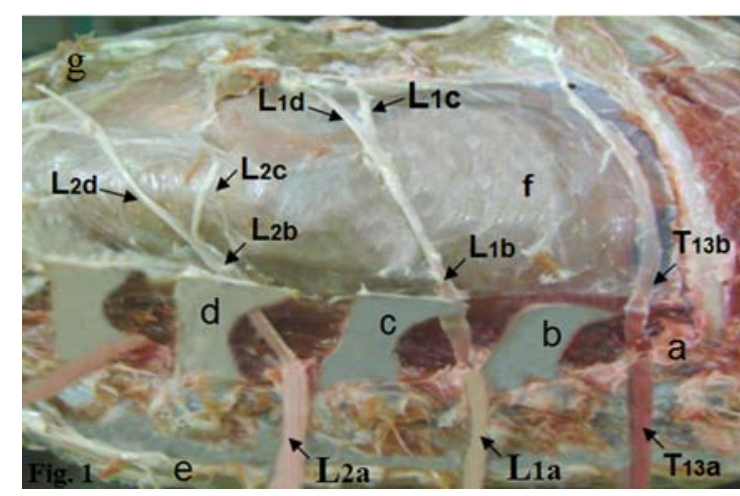

Fig.1: Branches of paravertebral spinal nerves and their landmarks. a: head of last rib, b: L1 transverse process, c: L2 transverse process, d: L3 transverse process, e: dorsal midline, f: peritoneum, g: abdominal muscles, T13a: dorsal branch of T13 spinal nerve, T13b: ventral branch of T13 spinal nerve, L1a: dorsal branch of L1 spinal nerve, L1b: ventral branch of L1 spinal nerve, L1c: medial branch of L1b, L1d: lateral branch of L1b, L2a: dorsal branch of L2 spinal nerve, L2b: ventral branch of L2 spinal nerve, L2c: medial branch of L2b, L2d: lateral branch of L2d.

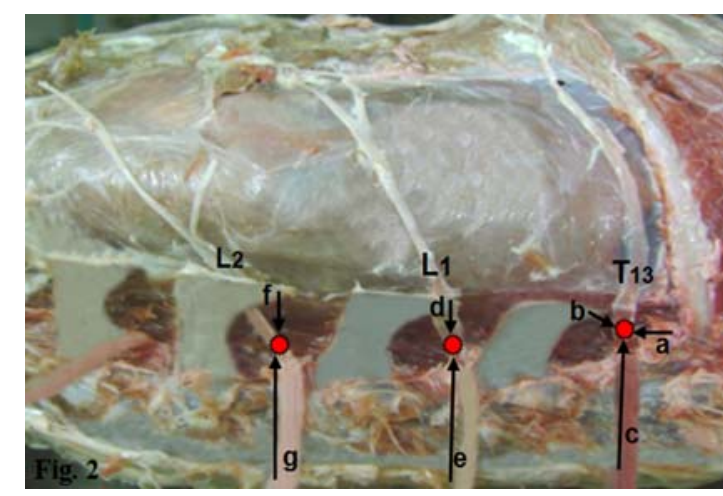

Fig.2: Sites of paravertebral spinal nerve block in vertical or proximal approach. T13: Last thoracic spinal nerve block (a: head of last rib to the nerve, b: L1 transverse process to the nerve, c: dorsal midline to the nerve), L1: First lumbar spinal nerve block (d: L2 transverse process to the nerve, e: dorsal midline to the nerve), L2: Second lumbar spinal nerve block (f: L3 transverse process to the nerve, g: dorsal midline to the nerve). 


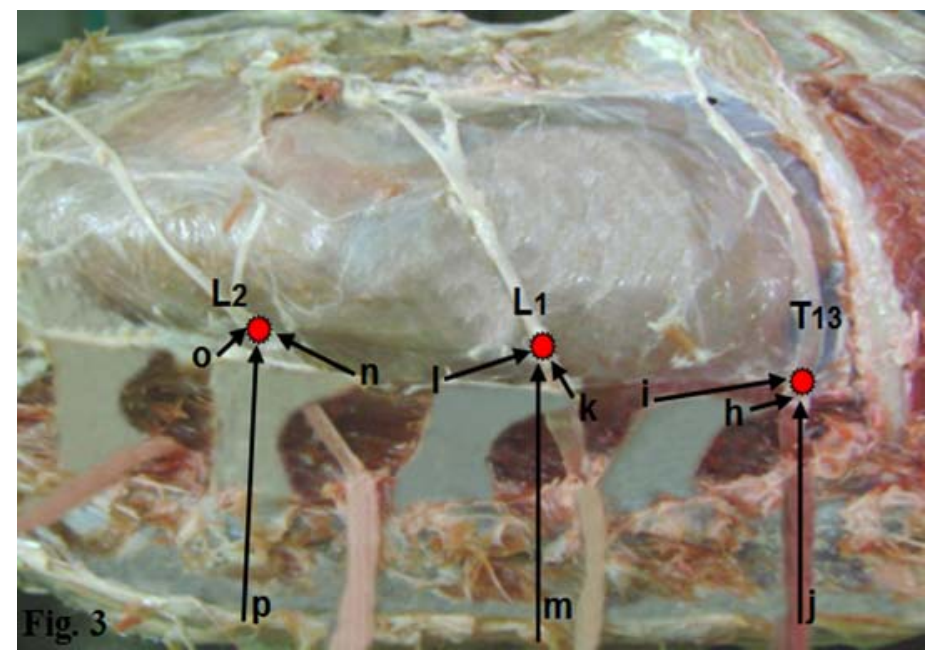

Fig.3: Sites of paravertebral spinal nerve block in horizontal or distal approach. T13: Last thoracic spinal nerve block (h: cranial angle of L1 transverse process to the nerve, i: caudal angle of L1 transverse process to the nerve, $\mathrm{j}$ : dorsal midline to the nerve), L1: First lumbar spinal nerve block (k: cranial angle of L2 transverse process to the nerve, l: caudal angle of L2 transverse process to the nerve, m: dorsal midline to the nerve), L2: Second lumbar spinal nerve block (n: cranial angle of L3 transverse process to the nerve, o: caudal angle of L3 transverse process to the nerve, p: dorsal midline to the nerve).

Proximal or vertical approach (Fig. 2)

Last thoracic spinal nerve

a: Head of last rib to the nerve: Head of the last rib to the nerve caudally, parallel to the dorsal midline.

b: First lumbar (L1) transverse process to the nerve: Cranio-lateral angle of L1 transverse process to the nerve cranio-medially.

c: Dorsal midline to the nerve: Dorsal midline to the nerve laterally at cranial border of L1 transverse process.

\section{First lumbar spinal nerve}

d: Second lumbar transverse process to the nerve: Cranio-lateral angle of L2 transverse process to the nerve medially.

e: Dorsal midline to the nerve: Dorsal midline to the nerve laterally at the cranial border of L2 transverse process.

\section{Second lumbar spinal nerve}

f: Third lumbar (L3) transverse process to the nerve: Cranio-lateral angle of L3 transverse process to the nerve medially.

g: Dorsal midline to the nerve: Dorsal midline to the nerve laterally at cranial border of the L3 transverse process.

\section{Distal or horizontal approach (Fig. 3)}

Last thoracic spinal nerve

h: Cranio-lateral angle of L1 transverse process to the nerve: Cranio-lateral angle of L1 transverse process to the nerve cranially.

i: Caudo-lateral angle of L1 transverse process to the nerve: Caudo-lateral angle of L1 transverse process to the nerve cranially.

j: Dorsal midline to the nerve: Dorsal midline to the nerve laterally at lateral border of L1 transverse process. 


\section{S. Sikder and others}

\section{First lumbar spinal nerve}

k: Cranio-lateral angle of L2 transverse process to the nerve: Cranio-lateral angle of L2 transverse process to the nerve caudally at lateral border of L2 transverse process.

l: Caudo-lateral angle of L2 transverse process to the nerve: Caudo-lateral angle of L2 transverse process to the nerve cranially at lateral border of L2 transverse process.

m: Dorsal midline to the nerve: Dorsal midline to the nerve laterally at lateral border of L2 transverse process.

\section{Second lumbar spinal nerve}

n: Cranio-lateral angle of L3 transverse process to the nerve: Cranio-lateral angle of L3 transverse process to the nerve caudally at lateral border of L3 transverse process.

o: Caudo-lateral angle of L3 transverse process to the nerve: Caudo-lateral angle of L3 transverse process to the nerve cranially at lateral border of L3 transverse process.

p: Dorsal midline to the nerve: Dorsal midline to the nerve laterally at lateral border of L3 transverse process.

\section{RESULTS AND DISCUSSION}

The last thoracic (T13) spinal nerve was located at $0.8 \pm 0.17 \mathrm{~cm}$ caudally from the head of the last rib, $0.7 \pm$ $0.22 \mathrm{~cm}$ cranio-medially from cranio-lateral angle of L1 transverse process and $3.4 \pm 0.20 \mathrm{~cm}$ laterally from dorsal midline in vertical or proximal approach. These measurements are clinically important to determine the exact site for the infiltration of local anaesthetic at the perineural area during paravertebral regional anaesthesia in black Bengal doe. Whereas the last thoracic spinal nerve is located at $3 \mathrm{~cm}$ off the dorsal midline at the cranial edge of L1 transverse process in caprine (Constantinescu, 2001), between the last rib and end of the L1 transverse process in ruminants(Kumar, 2003) and about 5 to $6 \mathrm{~cm}$ lateral to the mid-dorsal line behind the level of the head of the last rib in cattle (Venugopalan, 2000; Hall and Clarke, 1989), 2 inches lateral to the spinous process and off the anterior edge of the L1 transverse process in bovine (Riebold et al., 1982; Cakala, 1961).

The first lumbar (L1) spinal nerve was located at $1.2 \pm 0.40 \mathrm{~cm}$ cranially from the cranio-lateral angle of L2 transverse process and $3.5 \pm 0.17 \mathrm{~cm}$ laterally from the dorsal midline at cranial border of L2 transverse process and second lumbar (L2) spinal nerve was located at $1.6 \pm 0.26 \mathrm{~cm}$ cranially from the cranial angle of L3 transverse process and $3.7 \pm 0.14 \mathrm{~cm}$ laterally from the dorsal midline at cranial border of L3 transverse process (Table 1). These measurements are clinically important to determine the exact site for the infiltration of local anaesthetic at the perineural area during paravertebral regional anaesthesia in black Bengal doe. Whereas first (L1) and second (L2) lumbar spinal nerves are located at $3 \mathrm{~cm}$ off the dorsal midline at cranial edge of L2 and L3 transverse process in caprine (Constantinescu, 2001), between the transverse process of the L1 and L2, L2 and L3 vertebrae in ruminants (Kumar, 2003), about 5 to $6 \mathrm{~cm}$ lateral to the mid-dorsal line and behind the transverse process of L1 and L2 vertebrae in cattle (Venugopalan, 2000; Hall and Clarke, 1989) and 2 inches lateral to the spinous process and off the posterior edge of the transverse process of the L1 and L2 vertebrae in bovine (Riebold et al., 1982; Cakala, 1961). The variation in the measurement might be due to variation in the breed or age of the goat.

Table 1. Measurement from landmarks for the blocking sites of T13, L1 and L2 spinal nerve during paravertebral anaesthesia in proximal or vertical approach in black Bengal does $(\mathrm{n}=25)$

\begin{tabular}{|c|c|c|}
\hline Spinal nerves & Measurement from landmarks to the spinal nerves & Mean \pm SD $(\mathrm{cm})$ \\
\hline \multirow{3}{*}{$\mathrm{T} 13$} & Head of last rib to the nerve (a) & $0.8 \pm 0.17$ \\
\hline & First lumbar (L1) transverse process to the nerve (b) & $0.7 \pm 0.22$ \\
\hline & Dorsal midline to the nerve (c) & $3.4 \pm 0.20$ \\
\hline \multirow[t]{2}{*}{ L1 } & Second lumbar transverse process to the nerve (d) & $1.2 \pm 0.40$ \\
\hline & Dorsal midline to the nerve (e) & $3.3 \pm 0.17$ \\
\hline \multirow{2}{*}{ L2 } & Third lumbar (L3) transverse process to the nerve (f) & $1.6 \pm 0.26$ \\
\hline & Dorsal midline to the nerve (g) & $3.7 \pm 0.14$ \\
\hline
\end{tabular}


For distal or horizontal approach, the last thoracic spinal nerve (T13) was located at $0.5 \pm 0.10 \mathrm{~cm}$ cranially from cranio-lateral angle of the L1 transverse process, $1.8 \pm 0.63 \mathrm{~cm}$ cranially from caudo-lateral angle of L1 transverse process and $4.6 \pm 0.30 \mathrm{~cm}$ laterally from the dorsal midline at lateral border of L1 transverse process, while the blocking site of the last thoracic spinal nerve at the distal end of the L1 transverse process in caprine (Constantinescu, 2001), at posterior border of L1 transverse process in ruminants (Kumar, 2003) and in bovine to the tip of the L1 transverse process (Lee, 2006), beneath the transverse process of T13 vertebra in caprine (Hall and Clarke, 1989), at distal end of L1 vertebra in ruminants (Sharma, 2005).

The first lumbar (L1) spinal nerve was located at $0.4 \pm 0.10 \mathrm{~cm}$ caudally from cranio-lateral angle of the L2 transverse process, $1.4 \pm 0.28 \mathrm{~cm}$ cranially from caudo-lateral angle of L2 transverse process and $5.1 \pm 0.20 \mathrm{~cm}$ laterally from the dorsal midline at lateral border of L2 transverse process and second lumbar (L2) spinal nerve was found at $2.5 \pm 0.42 \mathrm{~cm}$ caudally from cranio-lateral angle of the L3 transverse process, $0.5 \pm 0.10 \mathrm{~cm}$ cranially from caudo-lateral angle of L3 transverse process and $5.5 \pm 0.28 \mathrm{~cm}$ laterally from the dorsal midline at lateral border of L3 transverse process (Table 2). These measurements are clinically important to determine the exact site for the infiltration of local anaesthetic at the perineural area during paravertebral regional anaesthesia in black Bengal doe. Where first (L1) and second (L2) lumbar spinal nerves are located at the distal end of the L2 and L4 transverse process in caprine (Constantinescu, 2001), at the distal end of the L2 and L3 vertebra in ruminants (Sharma, 2005), on the posterior border of the transverse process of L2 and L3 vertebra in ruminants (Kumar, 2003; Lee, 2006; Hall and Clarke, 1989). The variation in the measurement might be due to variation in the breed or age of the goat.

Table 2. Measurement from landmarks for the blocking sites of T13, L1 and L2 spinal nerve during paravertebral anaesthesia in distal or horizontal approach in black Bengal does $(\mathrm{n}=25)$

\begin{tabular}{|c|l|c|}
\hline Spinal nerves & Measurement from landmarks to the spinal nerves & Mean \pm SD $(\mathrm{cm})$ \\
\hline \multirow{4}{*}{ T13 } & Cranial angle of L1 transverse process to the nerve (h) & $0.5 \pm 0.10$ \\
\cline { 2 - 3 } & Caudal angle of L1 transverse process to the nerve (i) & $1.8 \pm 0.63$ \\
\cline { 2 - 3 } & Dorsal midline to the nerve (j) & $4.6 \pm 0.30$ \\
\hline \multirow{2}{*}{ L1 } & Cranial angle of L2 transverse process to the nerve (k) & $0.4 \pm 0.10$ \\
\cline { 2 - 3 } & Caudal angle of L2 transverse process to the nerve (l) & $1.4 \pm 0.28$ \\
\cline { 2 - 3 } & Dorsal midline to the nerve (m) & $5.1 \pm 0.20$ \\
\hline \multirow{3}{*}{ L2 } & Cranial angle of L3 transverse process to the nerve (n) & $2.5 \pm 0.42$ \\
\cline { 2 - 3 } & Caudal angle of L3 transverse process to the nerve (o) & $0.5 \pm 0.10$ \\
\cline { 2 - 3 } & Dorsal midline to the nerve (p) & $5.5 \pm 0.28$ \\
\hline
\end{tabular}

All the measurements from the landmarks of the specific spinal nerves are clinically important to determine the exact site for the deposition of anaesthetic at the perineural area during paravertebral regional anaesthesia in black Bengal doe.

In conclusion, this study revealed that for any surgical operation in the flank region performed easily by the perfect blocking of the T13, L1 and L2 spinal nerves. The measured parameters for T13, L1 and L2 spinal nerve from the specific landmarks should be considered during proximal or vertical and distal or horizontal approach of paravertebral regional anaesthesia for perfect deposition of local anaesthetic at the perineural area of the specific nerves and complete desensitization of all structures of the flank region in black Bengal doe.

\section{REFERENCES}

1. Cakala S (1961). A technique for the paravertebral lumbar block. Canadian Veterinary Journal, 7: $224-230$.

2. Constantinescu GM (2001). Paravertebral Lumbar (Nerve Block) Anaesthesia. In: Guide to Regional Anatomy Based on the Dissection of the Goat. $1^{\text {st }}$ Ed., Blackwell Publishing, USA, pp: 19-21.

3. Getty R (1975). Spinal Nerves, In: Sisson and Grossman's The Anatomy of the Domestic Animals, Vol 1 , $5^{\text {th }}$ Ed. ,W. B. Saunders Company, Philadelphia,USA. pp: 1135-1141.

4. Hossain M A (1984). Some aspects of gastro-esophageal reflux in anaesthetized sheep. Ph. D. Thesis, Royal (Dick) School of Veterinary Studies, University of Edinburgh, U. K. 


\section{S. Sikder and others}

5. Hall LW and Clarke KW (1989). Paravertebral Nerve Block. In: Veterinary Anaesthesia. $10^{\text {th }}$ Ed., Bailiere Tindall, London, pp: 322-324.

6. Hashim MA and Hossain MA (1989). Effects of starvation and positioning on gastro-esophageal reflux in anaesthetized goat during chloral hydrate-magnesium sulphate anaesthesia. Bang. J. Sci., pp: 62-69.

7. Lee L (2006). Local Anaesthesia \& Analgesia. In: Veterinary Surgery. Vol., 1, pp: 12-18http://instruction.cvhs.okstate. edu/vmed5412/pdf/14LocalAnaesthesia2006b.pdf

8. Kumar A (2003). Paravertebral Anaesthesia. In: Veterinary Surgical Techniques. $1^{\text {st }}$ Ed., Vikas Publishing House PVT. LTD., New Delhi,India, pp: 117-119.

9. Shokry M (1982). Regional Anaesthesia. In: Regional Anaesthesia in Buffaloes; A Review. $1^{\text {st }}$ Ed., Giza Publishing, Egypt, pp: 4.

10. Roe JM (1986). Bovine paravertebral analgesia: radiographic analysis and suggested method for improvement. Vet Rec., 119(10): 236-8.

11. Riebold TW, Goble DO and Geiser DR (1982). Large Animal Anesthesia Principles and Techniques. Animal Care and Use Committee. The Iowa State University Press. pp: 1-3.

12. Sharma SK (2005). Technique of Local Anaesthesia in Animals, pp: 11-13. http://hillagricernet.in/education/covas/ vsr/Anaesthesia/Anaesthesia\%20lectures/localanaesthesiatechniques.pdf.

13. Sloss V and Dufly JH (1977). Elective caesarean operation in Hereford cattle. Australian Veterinary Journal, 53(9): 420-4.

14. Venugopalan A (2000). Paravertebral Nerve Block In Bovine. In: Essentials of Veterinary Surgery. $8^{\text {th }}$ Ed., Oxford \& IBH Publishing Co. PVT. LTD, New Delhi, India. pp: 483-484. 